\title{
Mid Term Effects of a Single Injection versus Three Injections of Hyaluronic Acid in Patients with Rhizarthrosis
}

\author{
D. Perugia ${ }^{1,2}$, R. M. Lanzetti ${ }^{4}$, M. Vetrano ${ }^{1,3}$, C. Vavala ${ }^{1,3}$, S. Pascali ${ }^{1,3}$, S. M. Nusca ${ }^{1,3}$, F. \\ Santoboni ${ }^{1,3}$, M. C. Vulpiani ${ }^{1,3}$ \\ 1 Department of Surgical and Medical Sciences and Translational Medicine, Faculty of Medicine and Psychology, \\ Sapienza University of Rome, Rome, Italy \\ 2 Orthopedic Unit, Sant'Andrea University Hospital, Rome, Italy \\ 3 Physical Medicine and Rehabilitation Unit, Sant'Andrea University Hospital, Rome, Italy \\ ${ }_{4}^{4}$ Orthopedic and traumatology Unit, San Camillo Forlanini Hospital, Rome, Italy
}

\section{CORRESPONDING AUTHOR:}

Riccardo Maria Lanzetti

Orthopedic and traumatology Unit

San Camillo Forlanini Hospital

Rome, Italy

E-mail: riccardolanzetti@gmail.com

DOI:

10.32098/mltj.01.2020.18

LEVEL OF EVIDENCE: $2 b$

\begin{abstract}
SUMMARY
Background. To compare the effects of a single intra-articular injection of low molecular weight hyaluronic acid (LMWHA) versus 3 injections of the same LMWHA in patients affected by rhizarthrosis.

Methods. 40 patients received ultrasound (US) guided injections of LMW HA: Group A (20 patients) injections with $1 \mathrm{ml} \mathrm{HA}$; Group B (20 patients) once a week for 3 times injections of $1 \mathrm{ml} \mathrm{HA}$. Both groups were examined at baseline, at 1, 3 and 6 months follow-up using VAS (Visual Analogue Scale), Kapandji Thumb Opposition Test, DASH Score (functional assessment questionnaire) and DASH Work.

Results. Patients treated with a single HA injection had a statistically significant improvement $(\mathrm{p}<0.05)$ of the VAS at 1 - and 3-months follow-up compared to baseline, and of Kapandji thumb opposition test only at 6 months compared to baseline. Patients received 3 HA injections shown a statistically significant improvement $(\mathrm{p}<0.05)$ of the VAS, DASH score, DASH-work and Kapandji thumb opposition test at 1,3-and 6-months follow-up compared to baseline. The intergroup analysis did not underline any significant differences between the two groups at all follow-up.

Conclusions. The intra-articular HA injections allowed good effects in patients suffering from rhizarthrosis. To have a low term pain relief a single injection can be used. The 3 injections protocol is more indicated if we want to obtain also a functional improvement.
\end{abstract}

\section{KEY WORDS}

osteoarthritis; rhizartbrosis; Hyaluronic acid; infiltration

\section{BACKGROUND}

Osteoarthritis (OA) of trapeziometacarpal (TMC) joint also known as rhizarthrosis is a common condition primarily occurs in post-menopausal women (1). It concerns most frequently the non-dominant hand and causes pain and hand functional disability, including interference with grip and fine precision pinch (2). Rhizarthrosis is classified radiologically using either the Kellgren- Lawrence I-IV or Eaton and Glickel I-IV scales (3, 4).

Conservative management of TMC OA aims to provide pain relief and joint function improvement. It includes non-phar- macological treatments (local application of heat, therapeutic exercise, physical therapies, splints for thumb base OA, and orthoses) and pharmacological therapies (analgesic, corticosteroid injection, hyaluronic acid injection) (5).

According to the EULAR (European League Against Rheumatism) guidelines, the intra-articular injection with hyaluronic acid (HA) represents an effective conservative approach for patients suffering from rhizarthrosis (5). On the contrary, this therapy is not recommended by the ACR (American College of Rheumatology) experts (6). According to the SIR (Italian Society for Rheumatology) recommenda- 
tions, the treatment with hyaluronic injection can be indicated in patients with symptomatic TMC OA thank to his ability to reduce pain and improve functional limitation (7). HA represents the main constituent of the cartilage matrix in normal joints and improves lubrication. Different molecular weight (MW) exogenous HA are available for visco-supplementation: low (range: 500,000-730,000 Da), intermediate $(800,000-2,000,000 \mathrm{Da})$ and high MW (average: $6,000,000 \mathrm{Da}$ ) including cross-linked preparations (8).

In the last years few, authors assessed the efficacy of HA injections for the management of TMC OA (9-11).

The aim of this prospective study was to describe the effectiveness, in terms of improvement of pain and functionality, of a single intra-articular injection of low molecular weight (LMW) HA compared to a therapeutic protocol consisting of 3 injections of the same HA at 1, 3 and 6 months followup in patients affected by rhizarthrosis.

\section{MATERIAL AND METHODS}

This prospective, single-blinded, randomized clinical trial was performed in accordance with the Good Clinical Practice and the Declaration of Helsinki. The Institutional Ethics Review Board of our university hospital approved the study protocol. A placebo-controlled study was not allowed for ethical reasons. All patients were fully informed about the protocol of the study and gave written informed consent to participate, in accordance with the National Health Council Resolution No. 196/96.

From January 2016 to March 2017, patients with symptomatic TMC OA consecutively admitted to the outpatient clinic of Physical Medicine and Rehabilitation Unit of Sant'Andrea University Hospital of Rome ("Sapienza" University of Rome) were assessed.

Rhizarthrosis was graded according to Eaton and Glickel radiographic scale (Grade I: normal articular contours, joint space widening due to effusion or synovitis secondary to ligamentous laxity of basal joint; Grade II: joint space narrowing with some subchondral sclerosis, joint debris and osteophytes $<2 \mathrm{~mm}$; Grade III: complete loss of joint space, severe subchondral sclerosis, joint debris and osteophytes $>$ $2 \mathrm{~mm}$, scaphotrapezial joint is normal; Grade IV: changes found in stage III with the addition of scaphotrapezial joint narrowing and sclerosis) (4). Patients with grade II or III TMC OA, symptoms in the affected thumb for at least the 6 months prior to the start of the study, functional limitation during common daily living activities and a pain score VAS of at least 5 (range from 0 , absence of pain, to 10 , maximum pain imaginable) were eligible in the study. The exclusion criteria were: symptomatic bilateral disease, local or systemic infections, known allergy to HA, previous fractures, rheu- matic diseases, coagulopathies, intra-articular steroid injections within 6 months prior treatment, diagnosis of other conditions of the hand (such as, carpal tunnel syndrome, De Quervain syndrome, etc.) (figure 1).

Forty patients were enrolled in the study and were prospectively randomized to receive 1 or 3 intra-articular injections of LMW HA (Group A and Group B, respectively), using a computerized random number generator. Demographic and clinical features at baseline of the patients are summarized in table $\mathbf{I}$.

\section{INTERVENTIONS}

In both groups, it was used LMW HA (500-730 kDa, Hyalgan $20 \mathrm{mg} / 2 \mathrm{ml}$ Fidia Farmaceutici SpA). Group A (20 patients) received a single ultrasound (US) guided infiltra-

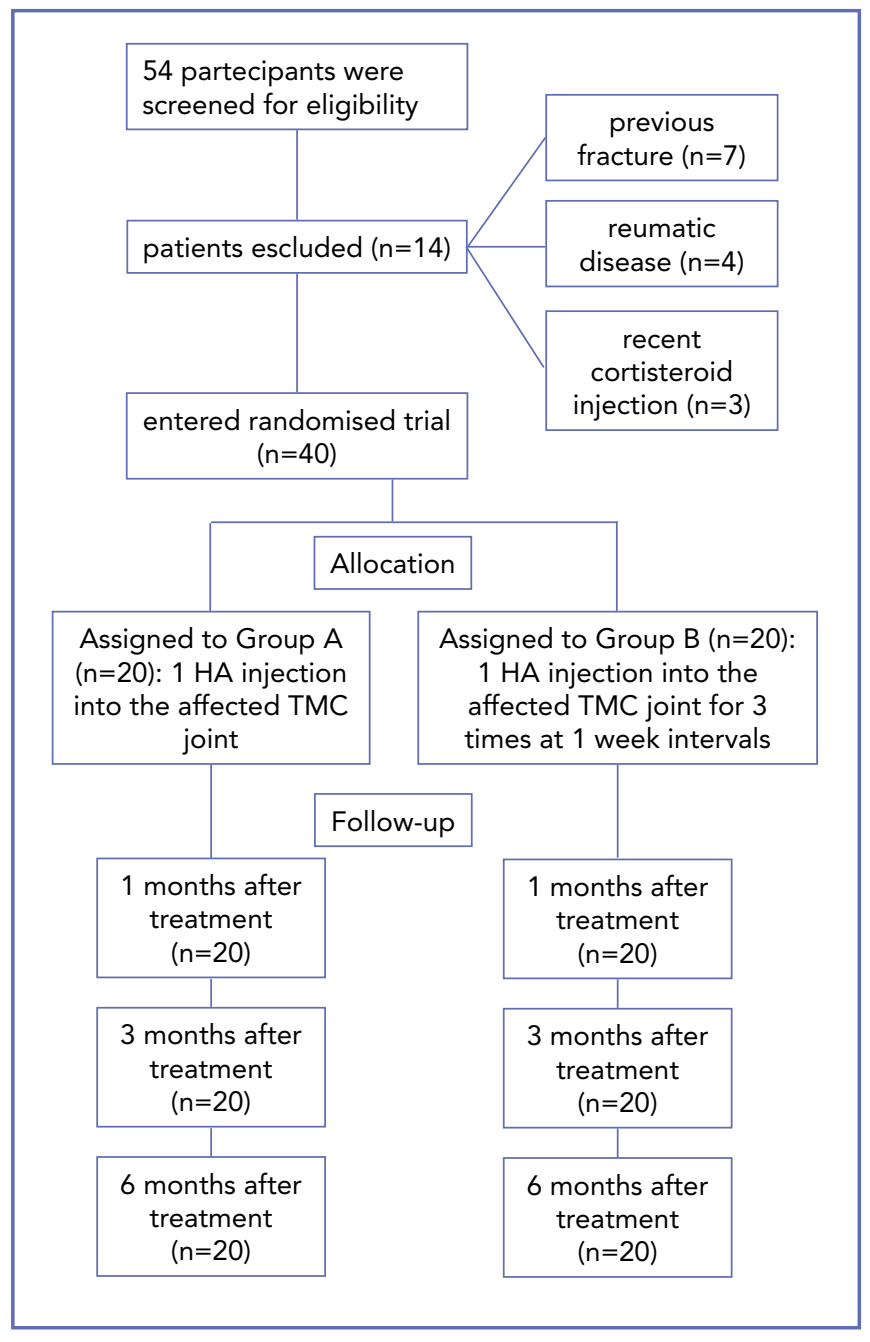

Figure 1. Consort diagram of the study. 
Table I. Demographic and clinical features.

\begin{tabular}{llll}
\hline Characteristics & $\begin{array}{l}\text { Group A } \\
\text { (1 HA } \\
\text { injections) }\end{array}$ & $\begin{array}{l}\text { Group B } \\
\text { (3 HA } \\
\text { injections) }\end{array}$ & $\begin{array}{l}\mathbf{p} \\
\text { value }\end{array}$ \\
\hline Patients & 20 & 20 & -- \\
\hline $\begin{array}{l}\text { Age, mean } \mathbf{H D}, \\
\text { (range), years }\end{array}$ & $\begin{array}{l}61.7 \pm 8.4 \\
(52-70)\end{array}$ & $\begin{array}{l}60.9 \pm 8.9 \\
(50-74)\end{array}$ & 0.980 \\
\hline Male/Female & $0 / 20$ & $4 / 16$ & 0.342 \\
\hline $\begin{array}{l}\text { Eaton and Glickel } \\
\text { (Grade II / III) }\end{array}$ & $14 / 6$ & $20 / 0$ & 0.060 \\
\hline
\end{tabular}

SD, standard deviation

tions with $1 \mathrm{ml} \mathrm{HA}$ into the affected TMC joint. Group B (20 patients) underwent US-guided injections with $1 \mathrm{ml} \mathrm{HA}$ once a week for 3 times into the affected TMC joint.

The US-guided injection were performed using a sonographic unit (Technos MP, Esaote ${ }^{\circledR}$, Genova, Italy) with a multi-frequency linear array probe $(7-12 \mathrm{MHz})$. Patients sat during the treatment with the forearm and hand in a neutral position. The ultrasound probe was placed longitudinally to the metacarpal axis on the dorsolateral side of the hand. The TMC joint space was identified laterally to the abductor pollicis longus tendon using a metal landmark positioned between the ultrasound probe and the skin of the patient and marked by a demographic marker. After disinfection and in aseptic conditions, the needle tip (22 Gauge) was placed under US-guidance on the marked line perpendicular to the skin. The capsular distension was a proof of intra-articular HA injection. After injection, the skin was disinfected and covered with medicated plaster. The patients were allowed to resume the common daily living activities immediately. The use of cryotherapy was advised as needed (1 application for 10-15 minutes) for pain control post-infiltration. Patients were not authorized to take NSAIDs or COX-2 inhibitors for pain relief during the study.

\section{OUTCOMES}

All patients were assessed by the same physician, blinded to the treatment group allocation, at baseline (T0), at 1 (T1), 3 (T2) and 6 (T3) months follow-up using following outcome measures: VAS (Visual Analogue Scale, range 0-10), Kapandji Thumb Opposition Test (score range: 0-10), DASH score (functional assessment questionnaire) and DASH work (12-14).

The VAS is a $10-\mathrm{cm}$ linear scale used to evaluate the intensity of pain (12). Patients had to mark the severity of pain from 0 labeled as "no pain" to 10 labeled as "the worst pain imaginable”.
The Kapandji Thumb Opposition Test is a measurement based on simple observation, which defines the ability to touch the four long fingers with the tip of the thumb. The score ranges from 0 to 10 . It is 1 for the lateral side of the second phalanx of the index finger, 2 for the lateral side of the third phalanx, 3 for the tip of the index finger, 4 for the tip of the middle finger, 5 for the ring finger and 6 for the little finger; moving the thumb proximally along the volar aspect of the little finger, the score is 7 when it touches the distal inter-phalangeal crease, 8 on the proximal inter-phalangeal crease, 9 on the proximal crease of the little finger and 10 when it reaches the distal volar crease of the hand (13).

The DASH Score is a 30 -items disability/symptom questionnaire used to assess the ability of a patient to perform certain upper extremity activities (14). The items regard the degree of difficulty in performing different physical activities due to: the arm, shoulder, or hand problem (21 items), the severity of each of the symptoms of pain, activity-related pain, tingling, weakness and stiffness (5 items), the problem's impact on social activities, work, sleep, and self-image (4 items). Each item has five response options which are used to calculate the DASH score ranging from 0 (no disability) to 100 (most severe disability).

The Dash Work module is used for patients whose disability is affecting their ability to work.

\section{STATISTICAL ANALYSIS}

The calculation of the sample size was performed considering a hypothetical difference between the two groups of 2 points in the VAS score at the T3 follow-up time point, with a standard deviation of 2 . The level of significance was set at alpha $=0.05$ and the power of the study at beta= 0.80 . The sample size required was 17 subjects per group, and 20 subjects per group were enrolled assuming a dropout rate of $15 \%$.

Continuous variables were summarized with mean and SD. Categorical variables were summarized by frequency and percentages. Difference between the two groups in patient's parameters at baseline were assessed with independent $t$ tests or $\chi^{2}$ tests. A p value $<0.05$ was considered as statistically significant. A two-way repeated measures ANOVA was run to determine the effect of the two treatments over time on pain (VAS) and function (DASH score and DASH work). Post-hoc analysis using the Bonferroni's test was used to determine the pairwise comparisons amongst the two treatment groups at all follow-up time points. The paired t-test was used for within-group comparisons. Changes in Kapandji Thumb Opposition Test during the follow-up period were analyzed with Friedman's test for intragroup 
analysis and with the Wilcoxon-Mann-Whitney test for between-group analysis. The level of statistical significance was set at $\mathrm{P}<0.05$.

Research is conducted according to international standards and as required by the journal as described in Padulo J. (15).

\section{RESULTS}

No patient reported local reactions or adverse effects to treatment. All patients completed the study and performed all follow-up visits.

Baseline demographic and clinical features of the patients are reported in table $\mathbf{I}$. The two groups were homogeneous at the basal time for age, gender, Eaton and Glickel grade, VAS, DASH score and DASH work; while statistically significant differences emerged for Kapandji Thumb Opposition Test (table II).

\section{VAS}

In Group A ( 1 injection) the mean VAS, that was $6.4 \pm 1.43$ at baseline, resulted as $4.00 \pm 2.67$ at 1 month, as $4.30 \pm 3.06$ at T2 and $4.70 \pm 2.83$ at T3, with statistically significant improvement at $1(\mathrm{p}=0.005)$ and 3 months follow-up compared to baseline ( $\mathrm{p}=0.002)$. Group B (3 HA injections) reported a reduction of the VAS at all follow-up $(7.60 \pm 1.43$

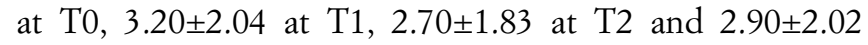

at T3), with a statistically significant improvement at 1 $(\mathrm{p}<0.001), 3(\mathrm{p}<0.001)$ and 6 months $(\mathrm{p}<0.001)$.

There were statistically significant time effects $\left(\mathrm{F}_{3,54}=31.43\right.$, $\mathrm{P}<0.001)$ and time*treatment effects $\left(\mathrm{F}_{3,54}=5.18, \mathrm{P}=0.003\right)$, but Bonferroni post hoc comparisons revealed not significant differences between the two treatment groups at all follow-up time points $(\mathrm{p}=0.927, \mathrm{p}=0.992, \mathrm{p}=0.749, \mathrm{p}=0.627$ respectively).

\section{KAPANDJI THUMB OPPOSITION TEST}

In Group A, the Kapandji Thumb Opposition Test was approximately constant over time with a value of $9.50 \pm 0.85$ at pre-treatment, $9.70 \pm 0.843$ at $\mathrm{T} 1,9.80 \pm 0.422$ at $\mathrm{T} 2$ and $9.90 \pm 0.316$ at $\mathrm{T} 3$, with a significant improvement only at T3 compared to T0 ( $\mathrm{p}=0.328, \mathrm{p}=0.147, \mathrm{p}=0.040$ respectively). In Group B, a statistically significant improvement was found at T1, T2, and T3 compared to baseline.

The analysis between the two groups reported a difference only at baseline $(\mathrm{p}=0.0001$ at $\mathrm{T} 0, \mathrm{p}=1.000$ at $\mathrm{T} 1$ and $\mathrm{T} 2$, $\mathrm{p}=0,481$ at T3).

\section{DASH SCORE}

In Group A, the mean DASH Score $(41.3 \pm 14.8$ at T0) was reduced at T1, T2 and T3 (mean 35.2 $\pm 18.3,29.1 \pm 12.9$, $33.4 \pm 12.7$ respectively) without any significant improve-

Table II. Statistical intergroup and intragroup analyses.

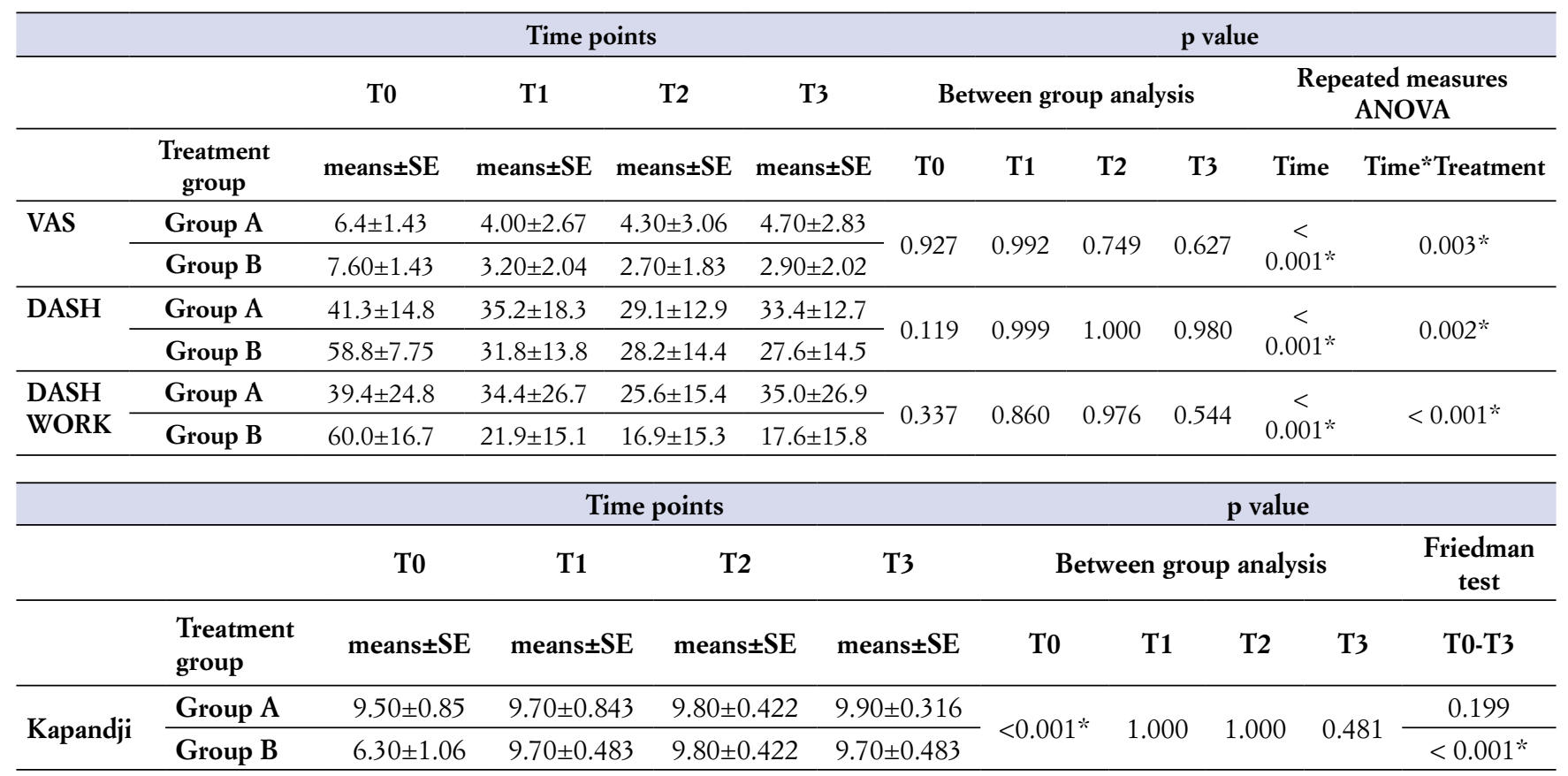


ment at all follow-up $(\mathrm{p}=0.869, \mathrm{p}=0.139, \mathrm{p}=0.655$ respectively). The Group B had a significant reduction of the DASH Score $(58.8 \pm 7.75$ at T0) at T1 $(31.8 \pm 13.8)$, T2 $(28.2 \pm 14.4)$ and T3 $(27.6 \pm 14.5)(\mathrm{p}<0.001$ at $\mathrm{T} 1$ and $\mathrm{T} 2$ and $\mathrm{p}<0.004$ at T3) compared to pretreatment.

There were statistically significant time effects $\left(\mathrm{F}_{354}=18.98\right.$, $\mathrm{P}<0.001)$ and time*treatment effects $\left(\mathrm{F}_{3,54}=5.59, \mathrm{P}=0.002\right)$, but not significant differences were described between the two treatment groups at all follow-up time points $(\mathrm{p}=0.119$, $\mathrm{p}=0.997, \mathrm{p}=0.999, \mathrm{p}=0.980$ respectively) by post hoc comparisons.

\section{DASH WORK}

In Group A, the mean DASH Work was $39.4 \pm 24.8$ at baseline, $34.4 \pm 26.7$ at 1 month, $25.6 \pm 15.4$ at 3 months and $35.0 \pm 26.9$ at 6 months without any statistically significant reduction compared to pretreatment $(\mathrm{p}=0.978, \mathrm{p}=0.166$, $\mathrm{p}=0.990$ respectively). For the Group B, a significant reduction $(p<0.001)$ of the DASH Work was found at all followup compared to baseline.

Statistically significant time effects $\left(\mathrm{F}_{3,54}=23.2, \mathrm{P}<0.001\right)$ and time*treatment effects $\left(\mathrm{F}_{3,54}=10.8, \mathrm{P}<0.001\right)$ were identified. However, by Bonferroni post hoc comparisons, not significant differences were found between the two treatment groups at all follow-up time points $(\mathrm{p}=0.337, \mathrm{p}=0.860$, $\mathrm{p}=0.976, \mathrm{p}=0.544$ respectively).

\section{DISCUSSION}

The aim of our clinical trial was to examine the effects of a single intra-articular injection (group A) of LMW HA compared to a therapeutic protocol consisting in 3 injections (group B) of the same HA at 1 (T1), 3 (T2) and 6 (T3) months follow-up in patients suffering from rhizarthrosis. Our results showed that both protocol treatments provided pain relief. Disability improvement was found mainly in patients who had received $3 \mathrm{HA}$ injections. Furthermore, Group B patients had better results over time showing a significant reduction of pain and disability in all follow-up compared to baseline; whereas in Group A it was found only an improvement of VAS at 1- and 3-months followup compared to baseline and of Kapandji Thumb Opposition Test only at T3 respect to T0. Nevertheless, no significant differences were found between the two groups for all outcomes evaluated.

Previous studies evaluated the efficacy of viscosupplementation for rhizarthrosis reporting promising results (9-11, 16-20). However, the comparison with the other trials present in the literature is often difficult due to the heterogeneity of protocol design, treatments (different type of HA, comparison with others intra-articular injection therapies) and time of follow-up used.

In a recent meta-analysis, it was underlined the ability of HA injection to increase functional capacity in patients affected by TMC OA (21).

On the contrary, Kroon et al. in their systematic review concluded that HA intra-articular injections are not more effective than placebo for this clinical condition discouraging the use of this treatment (22).

A randomized clinical study assessed the efficacy of HA compared to steroids injections in patients with grade II or III rhizarthrosis (16). Twenty patients underwent a single injection with $20 \mathrm{mg}$ triamcinolone acetonide. HA group (20 patients) received 1 sodium hyaluronate $(5 \mathrm{mg} / 0,5 \mathrm{ml})$ injection for three times at 1 week-intervals. All patients were evaluated before treatment and at 1, 3, 6 and 12 months after the first injection using the following parameters: VAS, pinch strength, grip strength and Duruöz Hand Index (DHI). A decrease of pain was seen at 12 months in steroid group and at 6 months in HA group. A grip strength improvement was found in both groups. Hand function increased significantly only in steroid groups. Our results are similar regarding pain relief. In addition, with respect to the mentioned trial, we reported an increase of hand functional capacity.

In 2009, Figen and collaborators studied the efficacy and tolerability of $\mathrm{HA}$ in patients with thumb base OA in a 6-months follow-up period (17). Thirty-three patients suffering from bilateral thumb base OA received Hylan G-F 20 injection in the TMC joint of a hand and saline-injection in the TMC joint of the other hand. VAS, pinch strength and functionality (Dreiser functional index) were evaluated at baseline, at 6th and 24th weeks. A statistically significant improvement was observed in all parameters at the 24th week in the Hylan G-F 20 group. Our clinical trial also reported pain reduction and disability improvement at a similar period follow-up. Notwithstanding, the comparison between the two studies is difficult because of the heterogeneity of protocol and HA molecular weight used.

Ingegnoli et al. aimed to evaluate the effects of high molecular weight HA US-guided intra-articular injection of TMC joints in OA (18). Sixty patients with bilateral symptomatic thumb base OA were treated bilaterally with 3 injections of high molecular weight $\mathrm{HA}$ at 1 -week intervals. All patients were clinically examined at baseline, after 2- and 24-weeks using VAS and Dreiser functional index. Furthermore, US examination was performed at baseline and during the follow-up period to assess TMC joints for synovial hypertrophy and Power Doppler Signal (PDS). An improvement of VAS and Dreiser's index was observed at 2 weeks and remains stable at 24 weeks follow-up. Power Doppler Signal decreased at 
2 weeks follow-up, but this data was not maintained at 24 weeks. This study presents some limitations: the lack of a control group and the small number of patients. Our clinical findings are similar to Ingegnoli's results. However, we think that the comparison between the 2 trials is not indicated owing to the use of different HA molecular weight.

A prospective case series study was performed to describe a simple and reliable procedure of US-guided injection with HA in TMC joints of patients affected by rhizarthrosis and to study the effects of viscosupplementation for the management of this clinical condition (19). Thirty-one patients were treated with 3 US guided-injections (at 1-week interval) with $1 \mathrm{ml}$ of HA. Patients sat with hand in the palmup position. The physician identified clinically the TMC joint and then performed US examination. A metal wire was positioned between the probe and the skin to identify TMC joint; then a demographic pencil was used to mark the wire profile. The US guided injection was performed using a 21-gauge needle that was positioned on the marked line perpendicularly to the probe, with an angle of $30-45^{\circ}$ to the skin. Furthermore, an independent radiologist performed US evaluation of TMC joint before and after injection to obtain baseline values and to detect joint effusion after injection. Patients were also assessed clinically at baseline and at 1,3 and 6 months after the first injection using the VAS and the Duruöz Hand Index (DHI). The US assessment showed a hyperechogenic distension of the capsule in all TMC joints treated, confirming a rate of accuracy of $100 \%$. A VAS reduction was found at 1 - and 3-months follow-up, while for the DHI scores no statistically significant difference was reported in all follow-up. A critical weakness of this study is the lack of a control group. Like these authors, we have also decided to use in our trial the US-guided infiltration to increase the accuracy of the procedure, showing the capsular distension in all patients. As the described study, we reported relief of pain (VAS) in patients underwent 3 HA infiltrations; however, with respect to this trial, our data shown, also, hand functional improvement. In their retrospective open-label study, Frizziero and collaborators assessed the efficacy of HA infiltrations to reduce pain and disability in 58 patients suffering from rhizarthrosis (grade II-III according to Kellgren Lawrence classification) (19). All patients were treated with an intraarticular infiltration of low molecular weight HA once weekly 3 times. At baseline and at 1,3-and 6-months after the treatment the following parameters were assessed: pain (VAS), NSAID intake, radial and palmar ab-/adduction, pinch strength. The authors reported a reduction of pain, hand mobility improvement, and NSAID's intake reduction compared to baseline. Similar data were found in our study in which we used the same protocol treatment in group B; indeed, we observed a significant improvement in pain and disability in patients receiving $3 \mathrm{HA}$ infiltrations. However, an important limitation of Frizziero's study is the lack of a control group.

In a randomized prospective trial, Monfort J et al. compared the efficacy and safety of ultrasound-guided intra-articular injections of hyaluronic acid versus betamethasone in the management of osteoarthritis of the thumb (9). In their study, 88 patients affected by OA of the thumb (Kellgren-Lawrence grade II-III) underwent 3 ultrasound-guided injections (at 7-day intervals) with low molecular weight HA or betamethasone. All patients were evaluated using the VAS, the Functional Index for Hand Osteoarthritis (FIHOA) scores and the Short Form-36 (SF-36) quality of life questionnaire at baseline and at 7, 14, 30, 90, and 180 days follow-up. In both treatment groups, it was found a significant reduction of VAS and Functional Index for Hand Osteoarthritis scores during follow-up at 7, 14, 30, 90, and 180 days compared to baseline. However, treatment with HA resulted more effective over time and it was especially indicated for patients with more severe symptoms. We agree with the results of this trial that it was conducted employing the same protocol ( $3 \mathrm{HA}$ injections, one per week) with a similar low molecular weight HA that we used in our study. More recently, Velasco et al. aimed to study the effects of viscosupplementation with Durolane (high molecular weight hyaluronic acid) in the treatment of rhizarthrosis (10). Thirty-five patients received a single injection of Durolane. The primary outcome used was the VAS. The secondary outcomes were: QuickDASH, Kapandji Thumb Opposition Test, radial abduction (flexion angle, measured in degrees), and metacarpophalangeal (MCP) joint flexion (measured in degrees) pinch (clamp) strength, the presence of crepitus, morning stiffness, and mobility difficulties. A significant relief of VAS and an improvement of other tests was found at 6 months after the infiltration. Our data also showed an improvement in pain and function in patients underwent HA injections. Nevertheless, we think that the comparison between the 2 trials is hard owing to the use of different protocols and HA types. Furthermore, Velasco et al. performed a single arm-study; we think that the presence of a control-group is essential to validate their findings.

In a retrospective study, Tenti and collaborators analyzed the clinical data of 100 patients with unilateral or bilateral TMC $\mathrm{OA}$, received 2 infiltrations of a hybrid formulation of HA (Sinovial H-L) or triamcinolone acetonide (Triamcinolone Group) (11). All patients were investigated at the time of the first and second injection and after one, 3- and 6-months using VAS and the Functional Index for Hand OA (FIHOA) as primary outcomes, and the duration of morning stiffness, the Health Assessment Questionnaire (HAQ) and the Medi- 
cal Outcomes Study 36-Item Short Form (SF-36) as secondary outcomes. Both therapies reported pain reduction and functional improvement. Nevertheless, the benefits were superior in Sinovial H-L group after 1 month and during the 6 months follow-up. Patients treated with Sinovial H-L had also a significant decrease in the duration of morning stiffness and an improvement in the HAQ score and physical component summary (PCS)-SF-36. Our clinical trial cannot be compared to this study because of the heterogeneity of methodology design and formulations HA used.

The present study describes the effects of HA in the management of TMC OA showing pain relief in both groups of treatment, and a greater functional improvement in patients treated with 3 injections. No differences were demonstrated between the two treatment protocols for VAS, Kapandji Thumb Opposition Test, DASH Score and DASH Work. Nevertheless, in patients treated with $3 \mathrm{HA}$ infiltrations we noticed better effects over the time. Indeed, a more lasting effect in pain relief was found in 3 HA protocol compared to $1 \mathrm{HA}$ treatment. Moreover, patients underwent 3 injections presented a major improvement of functional disability; probably this fact is due to the major viscosupplementation received by these patients and consequently to a bigger capsular distension.

The strengths of our study are his prospective nature and the use of ultrasound-guided infiltration. We are aware that

\section{REFERENCES}

1. Sodha S, Ring D, Zurakowski D, Jupiter JB. 2005 Prevalence of osteoarthrosis of the trapeziometacarpal joint. J Bone Joint Surg Am; 87:2614-8.

2. Zhang Y, Niu J, Kelly-Hayes M, et al. 2002 Prevalence of symptomatic hand osteoarthritis and its impact on functional status among the elderly: the Fra-mingham Study. Am J Epidemiol 156:1021-7.

3. Kellgren JH, Lawrence JS 1957 Radiological assessment of osteoarthrosis. Ann Rheum Dis. 16:494-502

4. Eaton RG, Glickel SZ. 1987 Trapeziometacarpal osteoarthritis. Staging as a rationale for treatment. Hand Clin 3:455-471.

5. Zhang W, Doherty M, Leeb BF, Alekseeva L, Arden NK, Biilsma JW, et al. 2007 EULAR evidence based recommendations for the management of hand osteoarthritis: report of a task force of the EULAR standing Committee for International Clinical Studies Including Therapeutics (ESCISIT). Ann Rheum Dis. 66:377-88

6. Hochberg MC, Altman RD, April KT, Benkhalti M, Guyatt G, McGowan J, et al. 2012 American College of Rheumatology 2012 recommendations for the use of non pharmacologic and pharmacologic therapies in osteoarthritis of the hand, hip, and knee. Arthritis Care Res (Hoboken). 64:465-74.

7. Manara M, Bortoluzzi A, Favero M et al. 2013 Italian Society for Rheumatology. Italian Society for Rheumatology our trial presents some limitations. First of all we studied a small number of patients. Another weakness is the heterogeneity of the patients at baseline regarding Kapandji thumb opposition test. For these reasons, the clinical relevance of our results may, potentially, be questioned.

This study suggests that HA can be considered a suitable and well tolerate conservative treatment for TMC OA. In our sample, the 3 injections protocol seems to be a better patient management options than 1 infiltration protocol. If we want to obtain low term pain relief we can resort to a single injection of HA. If we want to have also a functional improvement it is more indicated the $3 \mathrm{HA}$ protocol. Our findings must be confirmed by others clinical trials.

\section{ETHICS APPROVAL AND CONSENT TO PARTICIPATE}

All research conducted was in compliance with the Declaration of Helsinki and was approved by the IRB (Institutional Review Board) of "Sapienza” University and Sant' Andrea Hospital (Rome, Italy). All participants gave informed written consent at the start of the study.

\section{CONFLICT OF INTERESTS}

The authors declare they have no conflict of interests.

recommendations for the management of hand osteoarthritis. Reumatismo 65(4):167-85.

8. Maheu E, Rannou F, Reginster JY. 2016 Efficacy and safety of hyaluronic acid in the management of osteoarthritis: evidence from real-life setting trials and surveys. Semin Arthritis Rheum. 45(Suppl 4): S28-33.

9. Monfort J, Rotés-Sala D, Segalés N. 2015 Comparative efficacy of intra-articular hyaluronic acid and corticoid injections in osteoarthritis of the first carpometacarpal joint: results of a 6-month single-masked randomized study. Joint Bone Spine. 82(2):116-21.

10. Velasco E, Ribera MV, Pi J. 2017 Single-arm open-label study of Durolane (NASHA non animal hyaluronic acid) for the treatment of osteoarthritis of the thumb. Open Access Rheumatol. 9:61-66.

11. Tenti S, Pascarelli NA, Giannotti S, Galeazzi M, Giordano N, Fioravanti A. 2017 Can hybrid hyaluronic acid represent a valid approach to treat rizoarthrosis? A retrospective comparative study. BMC Musculoskelet Disord. 18(1):444.

12. Huskisson EC. Measurement of pain. (1974) Lancet. Nov 9;2 (7889):1127-31.

13. Kapandji A. Clinical test of apposition and counter-apposition of the thumb.1986 Ann Chir Main. 5(1):67-73.

14. Hudak PL, Amadio PC, Bombardier C. 1996 Development of an upper extremity outcome measure: the DASH (disabilities 
of the arm, shoulder and hand). The Upper Extremity Collaborative Group (UECG) Am J Ind Med. 29(6):602-8.

15. Padulo J., Oliva F., Frizziero A., Maffulli N. Muscles, Ligaments and Tendons Journal - Basic principles and recommendations in clinical and field Science Research: 2018 update. MLTJ 2018; 8(3): 305 - 307.

16. Bahadir C, Onal B, Dayan VY, Gürer N. 2009 Comparison of therapeutic effects of sodium hyaluronate and corticosteroid injections on trapeziometacarpal joint osteoarthritis. Clin Rheumatol. 28(5):529-33.

17. Figen Ayhan F, Ustün N. 2009 The evaluation of efficacy and tolerability of Hylan G-F 20 in bilateral thumb base osteoarthritis: 6 months follow-up. Clin Rheumatol. 28(5):535-41.

18. Ingegnoli F, Soldi A, Meroni PL. 2011 Power Doppler sonography and clinical monitoring for hyaluronic Acid treatment of rhizarthrosis: a pilot study. J Hand Microsurg. 3(2):51-4.
19. Di Sante L, Cacchio A, Scettri P, Paoloni M, Ioppolo F, Santilli V. 2011 Ultrasound-guided procedure for the treat-ment of trapeziometacarpal osteoarthritis. Clin Rheumatol. 30(9):1195-200.

20. Frizziero A, Maffulli N, Masiero S, Frizziero L. 2014 Six-months pain relief and functional recovery after intra-articular injections with hyaluronic acid (mw 500-730 KDa) in trapeziometacarpal osteoarthritis. Muscles Ligaments Tendons J. 4(2):256-61.

21. Trellu S, Dadoun S, Berenbaum F, Fautrel B, Gossec L. 2015 Intra-articular injections in thumb osteoarthritis: A systematic review and meta-analysis of randomized controlled trials. Joint Bone Spine 82:315-319

22. Kroon FPB, Rubio R, Schoones JW, Kloppenburg M. 2016 Intra-Articular Therapies in the Treatment of Hand Osteoarthritis: A Systematic Literature Review Drugs Aging 33:119-133. 